\title{
A model and case study for efficient shelf usage and assortment analysis
}

\author{
Mehmet Murat Fadılog̃lu • Oya Ekin Karaşan • \\ Mustafa Ç. Pınar
}

Published online: 13 December 2008

(C) Springer Science+Business Media, LLC 2008

\begin{abstract}
In the rapidly changing environment of Fast Moving Consumer Goods sector where new product launches are frequent, retail channels need to reallocate their shelf spaces intelligently while keeping up their total profit margins, and to simultaneously avoid product pollution. In this paper we propose an optimization model which yields the optimal product mix on the shelf in terms of profitability, and thus helps the retailers to use their shelves more effectively. The model is applied to the shampoo product class at two regional supermarket chains. The results reveal not only a computationally viable model, but also substantial potential increases in the profitability after the reorganization of the product list.
\end{abstract}

Keywords Retailing · Assortment optimization · Demand substitution

\section{Introduction}

One of the critical issues in Fast Moving Consumer Goods (FMCG) sector is the rapid pace of the business where new retail products are launched frequently. Firms operating in this sector are forced to adopt operational strategies that would enable them to keep up with the fast changing market conditions. At the retail store level, the new products have to share common shelf space with the already existing products. The sheer increase in the number of different products or SKUs (stock keeping units) causes product pollution in stores. On the other hand, the FMCG producers are looking for ways to increase their visibility through proliferating their SKUs to conquer shelf space. Both of these phenomena force retailers to

The authors gratefully acknowledge the support from the Turkish Academy of Science.

M.M. Fadılog̃lu (凶) • O.E. Karaşan · M.Ç. Pınar

Department of Industrial Engineering, Bilkent University, 06800 Ankara, Turkey

e-mail: mmurat@bilkent.edu.tr

O.E. Karaşan

e-mail: karasan@bilkent.edu.tr

M.Ç. Pınar

e-mail: mustafap@bilkent.edu.tr 
eliminate certain SKUs from their shelves in favor of others. However, the criteria used in this elimination/replacement are not entirely clear.

This study was initiated after we were approached by the local office of an international FMCG conglomerate. They complained that the product pollution, which is especially acute in the small and medium size retailers, was hurting their sales. They stated that these retailers are not aware of the phenomenon and its repercussions on their own profitability. Since the retailers were not taking any measures about the excessive number of SKUs they are carrying on their shelf space, they were also not allowing the products of this FMCG conglomerate to realize their full potential on this retail channel. They believed that the sales they were losing were more significant compared to other producers, since they were the market leaders in most product categories. The local office request was for us to develop a tool that the retailers could use to optimize their product mix on their shelves, which would also quantify the gain they can achieve by eliminating some SKUs. If the retailers implemented the recommendations of such a tool, this would reduce the product pollution and thereby, indirectly, benefit the sales of the conglomerate. The relation of the retailers with the conglomerate is a limited form of "category captainship" discussed in Kurtuluş and Toktay (2005).

Against this background the purpose of the present paper is to develop a viable optimization model which would prevent product pollution and simultaneously achieve an efficient assortment by judiciously deciding which products should find place on the retailer's shelves. The model does not consider how much shelf-space should be given to the products in the assortment, since in the studied retailers there are no well-defined shelf facings, and no available data to estimate the space elasticities. The model is applied to a specific hair care product class (shampoo) in two local chain stores and tested with actual data. The results summarized in the paper have been conveyed to the retail chains' managements and the resulting tool has been presented for their use.

Our contribution in this paper is an optimization model that improves the product mix on the shelf for any given product class at a retailer. There are many models in the literature with the same purpose. Yet, most models are complicated and have extensive data requirements. We claim that our model needs minimal data and can be used at/by almost any retailer that keeps track of their sales without complication. This model should be applicable to many smaller size retailers at which limited data is available.

The rest of this paper is organized as follows. In Sect. 2 we provide a brief overview of the related literature. Section 3 is devoted to the description and discussion of our model. A case study and results are reported in Sect. 4. Possible extensions and conclusions are given in Sect. 5.

\section{Literature review}

The literature in the area of assortment and shelf space management is quite large. We refer the readers to Mahajan and van Ryzin (1998), and Kök et al. (2005) for extensive reviews. Here we provide a brief review of the literature directly related to our manuscript.

An important work by Corstjens and Doyle (1981) provides an optimization model for shelf space allocation assuming a multiplicative self and cross space elasticity structure. They propose store experiments in order to estimate the parameters of their complicated elasticity structure. Since their estimation procedure is not practically viable, its applicability is limited. Inventory considerations are omitted in this model as is the case in ours. Yang and Chen (1999) develop an optimization model for shelf space allocation and describe how 
a decision support system for shelf space allocation can be implemented. Yang (2001) provides an efficient algorithm that retailers can use to decide on their shelf space allocation. Rajaram (2001) proposes a nonlinear integer programming model for the assortment selection problem in fashion retailing without considering space allocation as in the case of our model.

The two works in the literature most closely related to ours are Urban (1998) and Borin et al. (1994). Urban (1998) extends the model of Corstjens and Doyle (1981) so as to cover inventory and assortment considerations. He proposes an inventory-level-dependent demand structure, which quantifies the effect of shelf space allocation on the inventory dynamics. For the assortment considerations, he makes use of a linear multiplier which represents the probability of a customer switching from a product excluded from the assortment to another product. As a consequence of this linear approach to the substitution phenomenon, the extra demand for a given product due to switching from other products is lost if the product is taken out of the assortment. Combining all these elements, he proposes a complicated nonlinear programming formulation for the solution of which he suggests a greedy heuristic and a genetic algorithm. Borin et al. (1994) also propose a model that incorporates shelf space allocation, assortment and inventory effects. Due to the complicated nonlinear nature of their model, they are only able to provide a heuristic solution procedure based on simulated annealing. The assortment considerations in their work give rise to a substitution mechanism that is based on the same principle as the one we use in our work (that is discussed in Sect. 3.1). Both Urban (1998) and Borin et al. (1994) necessitate estimation of a prohibitive number of parameters such as cross space elasticities and switching probabilities for all product pairs. This limits viable application of the models especially when available data is scarce. Moreover, due to the complicated nature of the models they can only provide heuristic optimization procedures, whereas in our case we are able to guarantee an optimal solution in a reasonable amount of time as is demonstrated in Sect. 4 where we discuss the application of the model in a real retail setting.

A different and recent line of research on inventory and shelf space allocation is concerned with fresh produce. The evanescent nature of fresh produce renders the inventory control and shelf space allocation for this line of product challenging. The existing literature on perishable items typically consider lifetimes as random variables, yet neglect the effect of the utility decline over time; see Bai and Kendall (2008) for a brief survey. In a recent paper, Bai and Kendall (2008) address this issue by providing a model in which the utility of fresh produce continuously decreases over time. In the model, the demand rate is a function of both the displayed inventory level and the freshness of the produce. They optimize the shelf space allocation along with the inventory policy using a non-linear, integer programming model which the authors solve using a generalized reduced gradient (GRG) algorithm. In a subsequent paper, Bai et al. (2007) develop and implement several heuristic and meta-heuristic algorithms to solve larger instances of their model which cannot be handled efficiently with the GRG algorithm. In another paper more akin to our present work, Bai and Kendall (2005) propose a nonlinear integer model extending a multi-knapsack problem to decide on the most profitable allocation of shelf space to SKUs within a given product category. They do not incorporate cross space elasticities into their demand function mainly due to lack of reliable estimations. In our model, we consider the decision of keeping the product on or off the shelf. In this case, the cross-availability-elasticity which manifests itself as substitution cannot be ignored.

A critical part of our model is the assumption that the customers who cannot find the SKU of their preference will substitute that SKU with another one. In our study, we assume that substitution occurs within product categories. A version (without the categories) 
of such substitution can be observed in inventory models incorporating demand substitution (see Netessine and Rudi 2003 and references therein). Van Ryzin and Mahajan (1999) propose a newsboy model to determine the purchase quantities of different SKUs within a category. The paper makes use of a multinomial logit choice model to determine the individual purchase decisions. Each customer may choose to purchase among the listed products or may choose not to purchase anything. If the product of their choice in not available, the sale is lost. They show that the optimal assortment has a simple structure for their stylistic model in which the unit prices are identical. Mahajan and van Ryzin (2001) also allow dynamic substitution to occur when the SKU demanded is not in stock and use sample path analysis to obtain structural results. Smith and Agrawal (2000) investigate the effect of substitution on the optimal base-stock levels in multi-item inventory systems. Their formulation leads to an integer program. Cachon et al. (2005) also incorporate customer search into the choice model which is similar to the one in Van Ryzin and Mahajan (1999). Kök and Fisher (2006) provide an assortment optimization model that takes into account the effect of shelf space allocation on customer choice as well as the effect of inventory policy on stockout based substitutions.

\section{Model}

We group the products into categories according to their quality levels and prices. We assume $K$ product categories are available. For convenience of presentation we define the category set $\mathcal{K}=\{1, \ldots, K\}$ and the SKU set within each category $i \in \mathcal{K}, \mathcal{N}_{i}=\left\{1, \ldots, N_{i}\right\}$.

We make the following important modeling assumption: when a SKU is taken off the shelf an estimated portion of the demand for that SKU is distributed to other SKUs. This portion is determined according to a "substitution ratio" $s$. The specific computation of this parameter for our target application is described in detail in Sect. 4.

We will assume that the following data from the retail stores are available for our model:

- The SKU list for on-shelf products at each category.

- Sales data for a predetermined time horizon for each SKU in each category.

- Profit margins of each SKU in each category.

- Probability (frequency) of buying another SKU from the same retailer when a given SKU is not carried in that retailer.

The model is by nature static. We observe the average sales data once and decide on the optimal assortment with respect to it. The cost of keeping one SKU on the shelf per period is referred as $c$, and is assumed to be given and to be the same for all SKUs. We discuss the meaning of this parameter and its estimation in the following sections.

Our model aims to find the optimum SKU list to be kept on shelf at retailers so as to maximize the total average profit per period. Therefore, the goal is to decide which SKUs to eliminate from the list to minimize product pollution on the shelves while maximizing total profit. Now, we describe the ingredients of the model.

\section{Parameters:}

- $q_{i j}$ : average sales data per period for SKU $j$ of category $i$.

- $p_{i j}$ : profit obtained when one unit of SKU $j$ of category $i$ is sold.

- $c$ : cost per period of keeping one SKU on the shelf.

- $s_{i}$ : substitution ratio for category $i$. 
- $d$ : minimum ratio for directly satisfied demand at each category, referred to as the minimum conservation ratio.

\section{Decision variables:}

- The decision to keep SKU $i$ in category $j$ is modeled using a binary variable:

$$
x_{i j}= \begin{cases}1 & \text { SKU } j \text { of category } i \text { is kept in the list } \\ 0 & \text { otherwise. }\end{cases}
$$

\section{Constraints:}

- In our model, we have a single set of constraints to ensure that ratio of demand corresponding to undeleted SKUs to the total demand be no less than minimum conservation ratio $d$ for each category $i$ :

$$
\sum_{j=1}^{N_{i}} q_{i j} x_{i j} \geq d \sum_{j=1}^{N_{i}} q_{i j} \quad \text { for all } i \in \mathcal{K} .
$$

The optimization model for deciding the optimal SKU list that maximizes the total projected profit after substitution is:

$$
\begin{gathered}
\max Z \stackrel{\text { def }}{=} \sum_{i=1}^{K} \sum_{j=1}^{N_{i}} p_{i j}\left(q_{i j}+q_{i j} s_{i} \frac{\sum_{k=1}^{N_{i}}\left(1-x_{i k}\right) q_{i k}}{\sum_{k=1}^{N_{i}} x_{i k} q_{i k}}\right) x_{i j}-c \sum_{i=1}^{K} \sum_{j=1}^{N_{i}} x_{i j} \\
\text { s.t. } \quad \sum_{j=1}^{N_{i}} q_{i j} x_{i j} \geq d \sum_{j=1}^{N_{i}} q_{i j} \quad \text { for all } i \in \mathcal{K}, \\
x_{i j} \in\{0,1\} \quad \text { for all } i \in \mathcal{K}, j \in \mathcal{N}_{i} .
\end{gathered}
$$

\subsection{Discussion of the model}

Our first observation is that the optimization model (1)-(3) belongs to the class of nonlinear mixed integer programming problems. As such, it appears to be difficult to solve. However, as we shall see in Sect. 4, the linearized version of the model is solvable by off-the-shelf, state-of-the-art linear mixed-integer software packages for all dimensions that are of practical interest and of relevance to our case study.

The basic idea behind the model is that when a SKU disappears from a retailer's shelves, the demand for that SKU is not completely lost. Some of the customers who cannot find the specific SKU they are looking for switch to other SKUs. This phenomenon is known as "product substitution". Thus, although some profit is forgone when a SKU is "de-listed", there is also some new profit for other SKUs due to demand that switches. We assume that product substitution occurs within the SKUs in the same category. This means that a customer that would like to buy a specific premium shampoo, would only be willing to substitute it with another premium shampoo. The proportion of demand that stays in the retailer for category $i$ and switches to other products is a parameter of our model, $s_{i}$. This ratio is category dependent, since the substitution is more likely for low-end products, and less likely for premium products where brand loyalty is higher. Obviously, as $s_{i}$ increases, it is easier to unlist certain category $i$ items from the list since more of the demand is conserved and channeled to other SKUs. 
An additional point which relates to product substitution needs to be clarified in order to formulate a model. One needs to quantify how the substituting demand will be distributed among the products of the same category that are kept in the list. The assumption our model espouses is that this distribution will be according to the relative weight of sales for each SKU with respect to total sales corresponding to all SKUs that are not deleted from the list. This distribution principle is embedded into the model by taking the estimated sales after substitution for the SKU $j$ in category $i$ as

$$
q_{i j}+q_{i j} s_{i} \frac{\sum_{k=1}^{N_{i}}\left(1-x_{i k}\right) q_{i k}}{\sum_{k=1}^{N_{i}} x_{i k} q_{i k}} \quad \text { for all } i \in \mathcal{K} \text { and } j \in \mathcal{N}_{i}
$$

One should note that the ratio $q_{i j} /\left(\sum_{k=1}^{N_{i}} x_{i k} q_{i k}\right)$ represents the fraction of the sales of the SKU $j$ in category $i$ with respect to the total sales in category $i$ conserved by unlisting, and $\sum_{k=1}^{N_{i}}\left(1-x_{i k}\right) q_{i k}$ represents the total lost sales per period in category $i$ due to unlisting. Then $s_{i} \sum_{k=1}^{N_{i}}\left(1-x_{i k}\right) q_{i k}$ is the total substituting demand, which is the portion of the lost sales that is substituted with another product. When the substituting demand is multiplied with the fraction $q_{i j} /\left(\sum_{k=1}^{N_{i}} x_{i k} q_{i k}\right)$, one obtains the estimated additional sales in SKU $j$ in category $i$ due to substitution from the unlisted SKUs. Thus, our objective function consists of the difference of the total estimated profit after SKU elimination and the total cost of keeping the selected SKUs on the shelf as depicted in (1).

The substitution mechanism discussed above is based on the same principle as the one used in Borin et al. (1994), although the formulation they provide does not include the decision variables. This mechanism is the most viable way of modeling substitution with the data on hand. Another approach, which has been suggested in Urban (1998) is to determine the substitution coefficients between each pair of SKUs. Yet, it would be impossible in our setting to estimate all these coefficients as the retail managers, who were part of our study, also pointed out. Moreover, when this linear substitution mechanism is used, the extra demand for a given SKU due to switching from other SKUs-not in the list-is lost if that SKU is also taken out of the assortment. But, in reality a customer who is willing to substitute his/her choice would choose another product within the available assortmentnot outside of it.

It is reasonable to assume that the substitution ratios would not be dependent on the total quantity that is being substituted, since substitution is made by individual customers. When the proportion of the de-listed demand is not larger than $20 \%$ or $30 \%$, one does not have much reason to question this assumption. Yet, if this proportion is for example $90 \%$, it means that $90 \%$ of the demand would substitute to SKU's whose original demand is $10 \%$ of the total. Under such circumstances, the customer base would probably not be happy with the product mix presented on the retailer's shelves and the actual substitution ratio would be lower. Moreover, since such a policy of satisfying only a small portion of customers' original demand would be detrimental for the image of the retailer, it is reasonable to assume that the model should have a bound on the ratio of the substituting demand. The minimum conservation ratio in our model represents this bound on the ratio of substituting demand for each category, as expressed in (2). The constraint in (2) ensures that the model does not suggest solutions in the region where modeling assumptions begin to break.

A feature of the model worthy of discussion is that it does not involve the allocation of shelf space to the items in the assortment. Incorporating the shelf-space allocation would necessitate modeling the effect of shelf space allocation on the demand for the items on the shelves. This in turn would require estimation of cross space elasticities for each item pair under consideration. This model was applied in small and medium size retailers. These 
retailers keep minimal data which consists of monthly sales and price for each product. With the data available, it is not possible to estimate the effect of space used for the products. It is even hard to observe the space currently allocated, since there is no well-defined facings. Since they carry more SKUs than what they have space for, sometimes some of SKUs may be hidden behind others and the space allocation may change from day to day. These changes are not controlled by the management and are decided by the workers who replenish the shelves. Thus, although such an extension of the model would be theoretically possible, it would not be applicable to the setting under consideration.

Another critical component of our model is the cost of keeping SKUs on shelf, which is linear in the number of SKUs on shelf. In the small and medium size retailers where our study was concentrated, we observed that there were quite a few SKUs with very small sale volumes. With such small sale volumes, they should not be worth the effort of organizing them on the shelf, keeping track of their inventories etc. Moreover, we realized that if such SKUs could be eliminated there would be drastic reduction in terms of the product pollution. Thus, $c$ models all these indirect costs that are due to keeping one SKU on the list. Since this cost cannot be directly estimated by accounting methods, it has to be determined by asking the retail managers appropriate questions. It should be pointed out that such a setup cost per SKU in the assortment is also used in a similar study by Rajaram (2001). It is obvious that as $c$ increases, more elimination would take place and the optimal list would shrink.

As can be seen in (1), the cost components that correspond to each category are simply added up to constitute the objective function. Moreover, the constraints of the optimization model given in (2)-(3) only involve the decision variables of a single category at a time. Thus, our model can be decomposed into $K$ independent problems for each $i=1, \ldots, K$ as follows where we have also rewritten our objective function in a form that will suit better for the forthcoming analysis:

$$
\begin{gathered}
\max Z_{i} \stackrel{\text { def }}{=} \sum_{j=1}^{N_{i}}\left(\left(p_{i j} q_{i j}\left(1-s_{i}\right)-c\right) x_{i j}+s_{i} \sum_{k=1}^{N_{i}} q_{i k} \frac{p_{i j} q_{i j} x_{i j}}{\sum_{k=1}^{N_{i}} q_{i k} x_{i k}}\right) \\
\text { subject to } \sum_{j=1}^{N_{i}} q_{i j} x_{i j} \geq d \sum_{j=1}^{N_{i}} q_{i j}, \\
x_{i j} \in\{0,1\} \text { for all } j \in \mathcal{N}_{i} .
\end{gathered}
$$

We note that the original model (1)-(3) and the decomposed model (4)-(6) are both nonconvex and non-concave (neither quasi-convex nor quasi-concave) maximization problems over a set of integers. As such, they look quite difficult for numerical processing. Interestingly, a continuous relaxation that is immediately obtained by relaxing the integer (binary) requirement on the variables $x_{i j}$ does not yield a tractable problem either, in the sense that the resulting maximization problem is not guaranteed to have neither quasi-convex, nor quasi-concave objective function. To see this, let us observe that the decomposed problem (4)-(6) for category $i$ can be re-written in vector-matrix notation as

$$
\begin{array}{ll}
\max & Z_{i}=f^{\mathrm{T}} x+\frac{h^{\mathrm{T}} x}{q^{\mathrm{T}} x} \\
\text { s.t. } & q^{\mathrm{T}} x \geq d\left(e^{\mathrm{T}} q\right), \\
& 0 \leq x \leq e,
\end{array}
$$


where $f \in \mathbb{R}^{N_{i}}$ with component $j$ equal to $q_{i j} p_{i j}\left(1-s_{i}\right)-c, h \in \mathbb{R}^{N_{i}}$ with component $j$ equal to $s_{i} p_{i j} q_{i j} \sum_{k=1}^{N_{i}} q_{i k}, q \in \mathbb{R}^{N_{i}}$ with components $q_{i j}$, while $x \in \mathbb{R}^{N_{i}}$ denotes the vector with components $x_{i j}$, for all $j \in \mathcal{N}_{i}$, and $e \in \mathbb{R}^{N_{i}}$ is the vector of all ones. We note immediately that the continuous relaxation model above would be equivalent to a linear programming problem were it not for the presence of the term $f^{\mathrm{T}} x$ in the objective function. Without that term, the problem would fall into the realm of linear-fractional maximization with a quasi-convex and quasi-concave (hence, quasi-linear) objective function over a polyhedral set. It is well-known that such problems are easily transformed to equivalent linear programs. However, the presence of the term $f^{\mathrm{T}} x$ renders the objective function neither quasi-convex, nor quasi-concave in general (see Schaible 1977 for conditions regarding quasi-convexity or quasi-concavity of such functions). To see this, let us consider the level sets of the objective function

$$
S_{\alpha}=\left\{x \in \mathbb{R}^{N_{i}}: f^{\mathrm{T}} x+\frac{h^{\mathrm{T}} x}{q^{\mathrm{T}} x} \leq \alpha\right\}
$$

for some scalar $\alpha \in \mathbb{R}$. Notice that the positivity of the denominator is guaranteed from the constraint $q^{\mathrm{T}} x \geq d\left(e^{\mathrm{T}} q\right)$. Therefore, an equivalent expression for $S_{\alpha}$ is given by $\left\{x \in \mathbb{R}^{N_{i}}\right.$ : $\left.\left(q^{\mathrm{T}} x\right)\left(f^{\mathrm{T}} x\right)+h^{\mathrm{T}} x-\alpha q^{\mathrm{T}} x \leq 0\right\}$. Equivalently, we have

$$
S_{\alpha}=\left\{x \in \mathbb{R}^{N_{i}}: x^{\mathrm{T}}\left(\frac{f q^{\mathrm{T}}+q f^{\mathrm{T}}}{2}\right) x+h^{\mathrm{T}} x-\alpha q^{\mathrm{T}} x \leq 0\right\} .
$$

By definition of quasi-convexity, the set $S_{\alpha}$ should be a convex set. However, for this to hold true, one needs to guarantee positive semi-definiteness of the rank-2 matrix $\frac{f q^{\mathrm{T}}+q f^{\mathrm{T}}}{2}$, which is not true in general. A similar discussion with the sub-level sets $\left\{x \in \mathbb{R}^{N_{i}}: f^{\mathrm{T}} x+\frac{h^{\mathrm{T}} x}{q^{\mathrm{T}} x} \geq \alpha\right\}$ encounters the same conclusion.

Therefore, even the continuous relaxation of our model does not seem to be, at least from a theoretical point of view, an easy continuous optimization problem. Nonetheless, in the sequel, we shall reveal several interesting features of the nonlinear model along with a careful linearization that contribute to its numerical solution in reasonable running times. These features include a property of the local optima of the problem, bounds for preprocessing, the special case of uniform profit, and finally an effective linearization scheme.

\subsection{Local optima}

Consider the continuous relaxation of the decomposed model (4)-(6) where the constraint bounding the ratio of substituting demand for each category is eliminated. In particular, consider the following continuous optimization problem:

$$
\left(\mathrm{RP}_{i}\right) \quad \max \sum_{j=1}^{N_{i}}\left(\left(p_{i j} q_{i j}\left(1-s_{i}\right)-c\right) x_{i j}+s_{i} \sum_{k=1}^{N_{i}} q_{i k} \frac{p_{i j} q_{i j} x_{i j}}{\sum_{k=1}^{N_{i}} q_{i k} x_{i k}}\right)
$$

subject to $0 \leq x_{i j} \leq 1 \quad$ for all $j \in \mathcal{N}_{i}$.

Theorem 1 If $p_{i j} q_{i j}\left(1-s_{i}\right)-c>0 \forall j$, then any local optimal solution of $\mathrm{RP}_{i}$ is integral.

Proof Let $x_{i}^{*}=\left(x_{i 1}^{*}, x_{i 2}^{*}, \ldots, x_{i N_{i}}^{*}\right)$ be a local maximum of $\mathrm{RP}_{i}$. Without loss of generality assume that $0<x_{i l}^{*}<1$. We shall proceed to show that under the condition of this theorem, 
$x_{i}^{*}$ cannot be simultaneously as good as $x_{i}^{*}+\epsilon e_{l}^{\mathrm{T}}$ and $x_{i}^{*}-\epsilon e_{l}^{\mathrm{T}}$ where $e_{l}$ is the unit vector of dimension $N_{i}$ whose $l$ th component is 1 , and $\epsilon$ is a sufficiently small positive number that keeps these two vectors feasible. Assume to the contrary that $x_{i}^{*}$ is at least as good as both $x_{i}^{*}+\epsilon e_{l}^{\mathrm{T}}$ and $x_{i}^{*}-\epsilon e_{l}^{\mathrm{T}}$ in terms of objective value. In other words, we have

$$
\begin{aligned}
& \sum_{j} p_{i j} q_{i j} x_{i j}^{*}\left(1+s_{i}\left(\frac{\sum_{j} q_{i j}}{\sum_{j} q_{i j} x_{i j}^{*}}-1\right)\right)-c \sum_{j} x_{i j}^{*} \\
& \quad \geq\left(\sum_{j} p_{i j} q_{i j} x_{i j}^{*}+p_{i l} q_{i l} \epsilon\right)\left(1+s_{i}\left(\frac{\sum_{j} q_{i j}}{\sum_{j} q_{i j} x_{i j}^{*}+q_{i l} \epsilon}-1\right)\right)-c\left(\sum_{j} x_{i j}^{*}+\epsilon\right),
\end{aligned}
$$

and similarly,

$$
\begin{aligned}
& \sum_{j} p_{i j} q_{i j} x_{i j}^{*}\left(1+s_{i}\left(\frac{\sum_{j} q_{i j}}{\sum_{j} q_{i j} x_{i j}^{*}}-1\right)\right)-c \sum_{j} x_{i j}^{*} \\
& \quad \geq\left(\sum_{j} p_{i j} q_{i j} x_{i j}^{*}-p_{i l} q_{i l} \epsilon\right)\left(1+s_{i}\left(\frac{\sum_{j} q_{i j}}{\sum_{j} q_{i j} x_{i j}^{*}-q_{i l} \epsilon}-1\right)\right)-c\left(\sum_{j} x_{i j}^{*}-\epsilon\right) .
\end{aligned}
$$

After some manipulation, (9) is equivalent to:

$$
\begin{aligned}
& s_{i} \sum_{j} p_{i j} q_{i j} x_{i j}^{*}\left(\frac{q_{i l} \sum_{j} q_{i j}}{\sum_{j} q_{i j} x_{i j}^{*}\left(\sum_{j} q_{i j} x_{i j}^{*}+q_{i l} \epsilon\right)}\right) \\
& \quad \geq p_{i l} q_{i l}+p_{i l} q_{i l} s_{i}\left(\frac{\sum_{j} q_{i j}\left(1-x_{i j}^{*}\right)-q_{i l} \epsilon}{\sum_{j} q_{i j} x_{i j}^{*}+q_{i l} \epsilon}\right)-c .
\end{aligned}
$$

Similarly, (10) is equivalent to:

$$
\begin{aligned}
& s_{i} \sum_{j} p_{i j} q_{i j} x_{i j}^{*}\left(\frac{-q_{i l} \sum_{j} q_{i j}}{\sum_{j} q_{i j} x_{i j}^{*}\left(\sum_{j} q_{i j} x_{i j}^{*}-q_{i l} \epsilon\right)}\right) \\
& \quad \geq-p_{i l} q_{i l}-p_{i l} q_{i l} s_{i}\left(\frac{\sum_{j} q_{i j}\left(1-x_{i j}^{*}\right)+q_{i l} \epsilon}{\sum_{j} q_{i j} x_{i j}^{*}-q_{i l} \epsilon}\right)+c .
\end{aligned}
$$

Now, from (11) and (12) we have:

$$
\begin{aligned}
& \left(\sum_{j} q_{i j} x_{i j}^{*}\left(\sum_{j} q_{i j} x_{i j}^{*}+q_{i l} \epsilon\right)\right)\left(p_{i l} q_{i l}+p_{i l} q_{i l} s_{i}\left(\frac{\sum_{j} q_{i j}\left(1-x_{i j}^{*}\right)-q_{i l} \epsilon}{\sum_{j} q_{i j} x_{i j}^{*}+q_{i l} \epsilon}\right)-c\right) \\
& \quad \leq q_{i l} s_{i} \sum_{j} p_{i j} q_{i j} x_{i j}^{*} \sum_{j} q_{i j} \\
& \quad \leq\left(\sum_{j} q_{i j} x_{i j}^{*}\left(\sum_{j} q_{i j} x_{i j}^{*}-q_{i l} \epsilon\right)\right)\left(p_{i l} q_{i l}+p_{i l} q_{i l} s_{i}\left(\frac{\sum_{j} q_{i j}\left(1-x_{i j}^{*}\right)+q_{i l} \epsilon}{\sum_{j} q_{i j} x_{i j}^{*}-q_{i l} \epsilon}\right)-c\right)
\end{aligned}
$$

which is, after some simplifications, equivalent to

$$
p_{i l} q_{i l}\left(1-s_{i}\right)-c \leq 0,
$$

leading to a contradiction. 
Interestingly the quantity $p_{i j} q_{i j}\left(1-s_{i}\right)-c$ that decides the integrality of local optima in the relaxed problem also appears in the discussion of the uniform profit case (Sect. 3.4). This quantity is instrumental in solving the problem in the uniform profit case. While it is hard to give a precise economic interpretation to this quantity, we can view it as a modified profit coefficient.

\subsection{Bounds for pre-processing}

Now, we investigate lower and upper bounds on the change in the objective function value when we include a SKU in the list of SKUs on the shelf, while keeping everything else fixed. In other words, we assume to have a feasible vector $x$ and concentrate on the item $i, \ell$. We investigate the effect $\Delta Z_{i, \ell}$ on the objective function of making $x_{i, \ell}$ equal to one. Throughout this section, we use the decomposed model (4)-(6).

\section{Lemma 1}

$$
\begin{aligned}
\Delta Z_{i \ell}= & p_{i \ell} q_{i \ell}\left(1+s_{i}\left(\frac{\sum_{j} q_{i j}}{\sum_{j \neq \ell} q_{i j} x_{i j}+q_{i \ell}}-1\right)\right)-c \\
& -q_{i \ell} s_{i}\left(\frac{\left(\sum_{j} q_{i j}\right)\left(\sum_{j \neq \ell} p_{i j} q_{i j} x_{i j}\right)}{\left(\sum_{j \neq \ell} q_{i j} x_{i j}+q_{i \ell}\right)\left(\sum_{j \neq \ell} q_{i j} x_{i j}\right)}\right) .
\end{aligned}
$$

Proof After writing out the difference between the objective function values corresponding to the cases where $x_{i, \ell}=0$ and $x_{i, \ell}=1$, respectively, we obtain

$$
\begin{aligned}
\Delta Z_{i \ell}= & p_{i \ell} q_{i \ell}\left(1+s_{i}\left(\frac{\sum_{j} q_{i j}}{\sum_{j \neq \ell} q_{i j} x_{i j}+q_{i \ell}}-1\right)\right)-c \\
& +s_{i}\left(\sum_{j} q_{i j}\right)\left(\sum_{j \neq \ell} p_{i j} q_{i j} x_{i j}\right)\left(\frac{1}{\sum_{j \neq \ell} q_{i j} x_{i j}+q_{i \ell}}-\frac{1}{\sum_{j \neq \ell} q_{i j} x_{i j}}\right) .
\end{aligned}
$$

After some manipulation, Lemma 1 is obtained.

\section{Proposition 1}

$$
p_{i \ell} q_{i \ell}-c-q_{i \ell} \frac{s_{i}}{d}\left(\max _{j} p_{i j}\right) \leq \Delta Z_{i \ell} \leq p_{i \ell} q_{i \ell}\left(1+s_{i} \frac{1-d}{d}\right)-c-q_{i \ell} s_{i}\left(\min _{j} p_{i j}\right) .
$$

Proof From constraint (5) we know that

$$
\sum_{j} q_{i j} \geq \sum_{j} q_{i j} x_{i j} \geq d \sum_{j} q_{i j} .
$$

Therefore, we can give the following upper bound on $\Delta Z_{i \ell}$ :

$$
\Delta Z_{i \ell} \leq p_{i \ell} q_{i \ell}\left(1+s_{i}\left(\frac{\sum_{j} q_{i j}}{d \sum_{j} q_{i j}}-1\right)\right)-c-q_{i \ell} s_{i} \frac{\left(\sum_{j} q_{i j}\right)\left(\sum_{j \neq \ell} p_{i j} q_{i j} x_{i j}\right)}{\left(\sum_{j} q_{i j}\right)\left(\sum_{j \neq \ell} q_{i j} x_{i j}\right)} .
$$

Since $\sum_{j \neq \ell} p_{i j} q_{i j} x_{i j} \geq\left(\min _{j} p_{i j}\right) \sum_{j \neq \ell} q_{i j} x_{i j}$, we obtain:

$$
\Delta Z_{i \ell} \leq p_{i \ell} q_{i \ell}\left(1+s_{i} \frac{1-d}{d}\right)-c-q_{i \ell} s_{i}\left(\min _{j} p_{i j}\right) .
$$


Similarly, we can establish the lower bound

$$
\Delta Z_{i \ell} \geq p_{i \ell} q_{i \ell}-c-q_{i \ell} \frac{s_{i}}{d}\left(\max _{j} p_{i j}\right) .
$$

One can observe that the values of certain variables in the optimal solution can be determined by just checking the problem parameters based on the lower bound presented in the following proposition. This gives rise to the rule presented in Proposition 2. This rule is used for preprocessing the optimization model in order to reduce the problem size.

Proposition 2 If the lower bound on $\Delta z_{i \ell}$ is positive or zero, i.e.,

$$
p_{i \ell} q_{i \ell}-c-q_{i \ell} \frac{s_{i}}{d}\left(\max _{j} p_{i j}\right), \geq 0
$$

then $S K U \ell$ of category $i$ is guaranteed to be in an optimal list, i.e., we can set $x_{i \ell}=1$ without loss of optimality.

This proposition stipulates that the SKU $\ell$ in category $i$ should be kept in the list, if the corresponding lower bound is greater or equal to zero. This is due to the fact if this SKU is added to the list the objective function will improve by at least the value of the lower bound, irrespective of the composition of the list. Since adding an additional SKU to the list cannot violate any constraint of the program, we are never worse off when that SKU is in the list.

One can think of a similar proposition using the upper bound which would stipulate that a SKU should be kept off the list if the corresponding upper bound is nonpositive. Yet, the problem is that by keeping a SKU out of the list one can violate constraint (5). Thus, such an elimination can only be done if this constraint is nonbinding at the optimal point. Since this requires post-optimization processing, its use in pre-processing would be complicated. Still if constraint (5) is superfluous, i.e., $d=0$, then the upper bound would also yield a pre-processing rule.

\subsection{A special case: uniform profit}

In this section, we present how the model simplifies when the profit margins are equal for each SKU within a category, i.e. when $p_{i j}=p_{i}$ for all $j=1, \ldots, N_{i}$. Under this condition the objective function of the decomposed problem given in (4), would simplify to

$$
Z_{i}=\sum_{j}\left(p_{i}\left(1-s_{i}\right) q_{i j}-c\right) x_{i j}+p_{i} s_{i} \sum_{j} q_{i j} .
$$

If it were not for the constraint (5), in this setting the optimization problem would decompose to $N_{i}$ independent subproblems involving one SKU at a time. This gives rise to the next proposition where $x^{*}$ denotes an optimal solution and $x^{c}$ denotes a candidate solution. Let $\Delta_{i j}^{u}=p_{i}\left(1-s_{i}\right) q_{i j}-c$.

\section{Proposition 3}

1. For each $j \in \mathcal{N}_{i}$ if $\Delta_{i j}^{u} \geq 0$ then set $x_{i j}^{c}=1$, otherwise set $x_{i j}^{c}=0$.

2. If $\sum_{j} q_{i j} x_{i j}^{c} \geq d \sum_{j} q_{i j}$ then $x_{i j}^{*}=x_{i j}^{c}$.

3. If $\sum_{j} q_{i j} x_{i j}^{c}<d \sum_{j} q_{i j}$ then use the following procedure: 
(a) Sort those indices $j \in \mathcal{N}_{i}$, in descending order according to the quotient $\Delta_{i j}^{u} / q_{i j}$. Let $j_{l}$ denote the corresponding index in the original list of the lth element in the sorted list.

(b) Find $l^{*}$ as the minimum $l$ satisfying $\sum_{m=1}^{l} q_{i j_{m}} \geq d \sum_{j=1}^{N_{i}} q_{i j}$.

(c) Set $x_{i j_{l}}=1$ for $l=1, \ldots, l^{*} ; x_{i j_{l}}=0$ for $l=l^{*}+1, \ldots, N_{i}$.

The proposition states that the candidate solution based on the optimal solutions of the $N_{i}$ subproblems would be optimal for the overall problem for category $i$, if constraint (5) is satisfied. In the case where constraint (5) is violated by the candidate solution, one needs to set a larger number of variables to one, which is achieved by means of the simple procedure described in the proposition. In the latter case, one deals essentially with a reverse knapsacktype problem, and the proposed procedure is typical for this class of problems.

\subsection{Linearization}

To compute an optimal solution to the non-convex, nonlinear formulation (4)-(6), we develop an efficient linearization scheme. Recall that

$$
Z_{i}=\sum_{j=1}^{N_{i}}\left(\left(p_{i j} q_{i j}\left(1-s_{i}\right)-c\right) x_{i j}+s_{i} \sum_{k=1}^{N_{i}} q_{i k} \frac{p_{i j} q_{i j} x_{i j}}{\sum_{k=1}^{N_{i}} q_{i k} x_{i k}}\right) .
$$

We shall force variable $y_{i}$ take on the value $\frac{\sum_{j} p_{i j} q_{i j} x_{i j}}{\sum_{j} q_{i j} x_{i j}} \forall i \in \mathcal{K}$. If the denominator $\sum_{j} q_{i j} x_{i j}>0$ (a condition guaranteed by (5)), the above relation can be forced with the equation:

$$
\sum_{j} p_{i j} q_{i j} x_{i j}=\sum_{j} q_{i j} y_{i} x_{i j}, \quad i \in \mathcal{K}
$$

Let

$$
z_{i j}=y_{i} x_{i j} \quad i \in \mathcal{K}, j \in \mathcal{N}_{i}
$$

Proposition 4 Let $M_{i}=\max _{j} p_{i j}$ for all $i \in \mathcal{K}$. Then, for each $i \in \mathcal{K}$ the following model correctly linearizes the original decomposed model (4)-(6):

$$
\begin{gathered}
\max \sum_{j}\left(p_{i j} q_{i j}\left(1-s_{i}\right)-c\right) x_{i j}+s_{i} \sum_{j} q_{i j} y_{i} \\
\text { subject to: } \quad \sum_{j} p_{i j} q_{i j} x_{i j}=\sum_{j} q_{i j} z_{i j}, \quad j \in \mathcal{N}_{i}, \\
z_{i j} \leq y_{i}, \quad j \in \mathcal{N}_{i}, \\
z_{i j} \geq y_{i}-M_{i}\left(1-x_{i j}\right), \quad j \in \mathcal{N}_{i}, \\
z_{i j} \leq M_{i} x_{i j}, \quad j \in \mathcal{N}_{i}, \\
\sum_{i} q_{i j} x_{i j} \geq d \sum_{j=1}^{N_{i}} q_{i j}, \quad j \in \mathcal{N}_{i}, \\
x_{i j} \in\{0,1\}, \quad j \in \mathcal{N}_{i} .
\end{gathered}
$$


Proof We are going to use the classical big- $M$ linearizations of equations (15). Indeed, we would like to force the following relationships:

$$
z_{i j}=\left\{\begin{array}{ll}
y_{i} & \text { if } x_{i j}=1, \\
0 & \text { if } x_{i j}=0,
\end{array} \quad i \in \mathcal{K}, j \in \mathcal{N}_{i},\right.
$$

which are equivalent to (15).

For $M_{i}$ large enough, inequalities (18)-(20) force relationship (23). If $x_{i j}=1$ then inequalities (18) and (19) force $z_{i j}=y_{i}$, where inequality (20) becomes a redundant constraint. On the other hand, if $x_{i j}=0$, then inequality (20) forces $z_{i j}=0$ where both (18) and (19) become redundant constraints. For these linearizations to work properly $M_{i}$ should be at least as large as the maximum value each $y_{i}$ and consequently each $z_{i j}$ can take. An appropriate such value for $M_{i}$ is found from the following inequality:

$$
y_{i}=\frac{\sum_{j} p_{i j} q_{i j} x_{i j}}{\sum_{j} q_{i j} x_{i j}} \leq \max _{j} p_{i j} \frac{\sum_{j} q_{i j} x_{i j}}{\sum_{j} q_{i j} x_{i j}}=M_{i} .
$$

To strengthen the linear model (16)-(22), we also added the following valid inequality for each $i \in \mathcal{K}$ to our model

$$
\sum_{j=1}^{N_{i}} p_{i j} q_{i j} x_{i j} \geq y_{i} d \sum_{j=1}^{N_{i}} q_{i j}
$$

which proved very effective in our numerical experiments.

\section{Case study at two local chains}

We developed our model upon the request of a major international FMCG conglomerate which has significant presence in the Turkish market. The company has the largest market share in Turkey in its core operating sectors: Fabric and Home Care products, Health and Beauty Care products, Feminine Care products and Baby Care products. The company wanted us to provide a tool that small and medium size supermarkets in Turkey could use to optimize the assortment on their shelves. They believed that if the supermarkets would eliminate the unprofitable SKUs from their shelves, the visibility of their own products, which are usually the product leaders, would increase and thereby, their sales would be positively affected. Under such a scenario, the provided tool would assist the creation of a win-win situation for both the supermarket and the FMCG conglomerate. They shared their intentions with two local supermarket chains in Ankara, Turkey which in return accepted to collaborate with us. These supermarket chains are considered as part of the "Local chain" customer channel that constitutes $35 \%$ of the FMCG company's total sales volume in the country.

In this case study, we discuss the results of our model on the assortment of shampoo products at two local chains in the Ankara region. Since these two supermarkets were only keeping monthly sales and profitability data, our model was originally shaped around these input requirements. The two chains agreed to disclose the requisite data for the purposes of the project. Although they stated that they could use such a tool for different product classes, they first wanted us to demonstrate the effectiveness of our methodology in a specific product class. We decided to focus on the shampoo products since this product class has the maximum number of SKUs on retailers' shelves while frequent new product launches are 
still pushing the number higher. Therefore, the choice of shampoo class is definitely one where efficient assortment and shelf usage is to be beneficial. Moreover, since the FMCG company has a significant presence in this class, they agreed with this choice which could help increase the visibility of their market leader products.

The study concentrates on two local chains, that will be referred to henceforth as "Local chain A" and "Local chain B", and on three stores of each local chain classified according to their sizes as "small", "medium" and "large". The shampoo SKUs under scrutiny are classified into three categories according to their quality levels and prices, the premium quality in category 1 , the middle quality in category 2 , and low quality in category 3.

After consulting with retail managers we decided that setting the cost per month of keeping a SKU on the shelf, parameter $c$ in our model, to 1 local currency unit (YTL) is a reasonable choice. We investigate the sensitivity of our results to perturbations in this value in Experiment 3 below.

A preliminary analysis of sales data according to categories, brands and store sizes yielded the following results:

- Although some SKUs are sold in smaller quantities, they bring larger profit.

- Results change according to store size.

- There exist seasonal effects in sales; especially during the summer months noticeable increases in sales of shampoo occur.

- There are promotions in some SKUs affecting the sales figures.

- Prices may fluctuate over months.

The SKUs with small sales yet with large profit are always kept in the assortment by our optimization model. The model actually tries to increase the sale figures of these SKUs by deleting some unprofitable items from the assortment. These SKUs experience additional demand from the customers who switch to products available in the assortment. Obviously, this substitution is quite favorable for the retailer's profit.

The local chain managers reconsider the SKUs to be carried on their shelves twice a year. As a result, we decided to use data corresponding to six calender months each time an instance of the model is solved. But, in order to accommodate the seasonal effects in demand, the data of each six months period is employed to decide on the assortment of the next period corresponding to the same months of the year (using the data with a lag of six months). This means we use the data of the previous period corresponding to the same season for the current assortment. Average prices for SKUs were used in our calculations to eliminate possible price fluctuations that may have occurred during the six month period. Note that the six month period is long enough for the averages to incorporate both promotion and regular price epochs.

It should be noted that the model we propose only deletes SKUs from the current assortment. It does not have a feature to incorporate new SKUs that may become available in time, or old SKUs whose popularity may rise due to new marketing efforts. In order to accommodate the situation, we propose the chains to add the SKUs that look promising to their assortment for a period of six months. After that our methodology would take care of the decision of keeping them on shelves or delisting them.

One should also note that as the excessive number of SKUs carried at the retailer's shelves decreases to reasonable levels due to our model, the product pollution would decrease. This would allow the retailer to reorganize its shelves in a more orderly and attractive fashion. Thus, we would actually expect this decrease to give rise to some additional demand from visiting customers who may have been previously overwhelmed by the sheer number of available SKUs and product pollution, and consequently have left the shop without buying any shampoo. This model does not take into account this additional demand 
since it cannot be predicted based on the sales and profitability data available without observing displayed inventory levels. This only means that under more realistic considerations, we would eliminate even more SKUs and that the estimated profit in the objective function would be greater.

\subsection{Calculation of the substitution ratio}

We observed that if a desired SKU is out of stock, a fraction of the demand for that particular SKU will be distributed among other shampoos of the same category. This switching behavior is quantified using a parameter $s$ that we refer to as the substitution ratio. According to a marketing survey commissioned upon the request of the FMCG company, a customer who cannot find his/her desired shampoo:

(a) buys another brand with a probability of 0.16

(b) leaves the store without buying shampoo with a probability of 0.10

(c) buys the same SKU from another store with a probability of 0.53

(d) buys another SKU of the same brand with a probability of 0.15

(e) delays shopping with a probability of 0.06

Similar findings are also reported in Corsten and Gruen (2003). Our main observation here is that the substitution ratio is stable within a product category. That is to say, when a customer comes to buy a relatively cheaper product (third quality in our particular case study) and faces an out-of-stock situation, he/she buys another product in the same category as the one he/she was looking for. However, a customer looking for a first quality shampoo will be less likely to switch to a lower quality shampoo when his/her preferred shampoo is not available. Unfortunately, we have no access to data to estimate different substitution ratios for different quality categories. Therefore, we decided to work with a single aggregate substitution ratio estimate valid for all quality classes. This aggregate estimate was calculated as follows using the previously mentioned marketing survey. We made the assumption that customers will keep using shampoo for hair care in the long run, and those customers "buying nothing" in the group b. above will come back to the same store with a 0.5 probability. Adding to this figure, the probabilities from a, $d$ and e groups above, we obtain the estimate 0.42 for $s$ used in the rest of this study. We also provide numerical results below for the sensitivity of our findings to the particular value of the substitution ratio.

\subsection{Results}

We used GAMS/CPLEX 10 system to solve the linear optimization model introduced in Sect. 3.5 to optimality for each of the three stores of Local chain A and those of Local chain B. We summarize below our results in three experiments.

Experiment 1 The impact of the minimum conservation ratio. In Tables 1 and 2 we report the results of our computational experiments for both Local chain A and Local chain B, respectively, with the substitution ratio $s$ and cost parameter $c$ fixed, under three different values of the minimum conservation ratio $d$, namely $0.6,0.8$ and 0.9 . The first column under the heading "Initial obj" gives the initial objective function value (the difference between the total estimated profits and SKU keeping costs) using the data made available for this study. Under the column "Optimal obj" we report the value of the objective function after the 


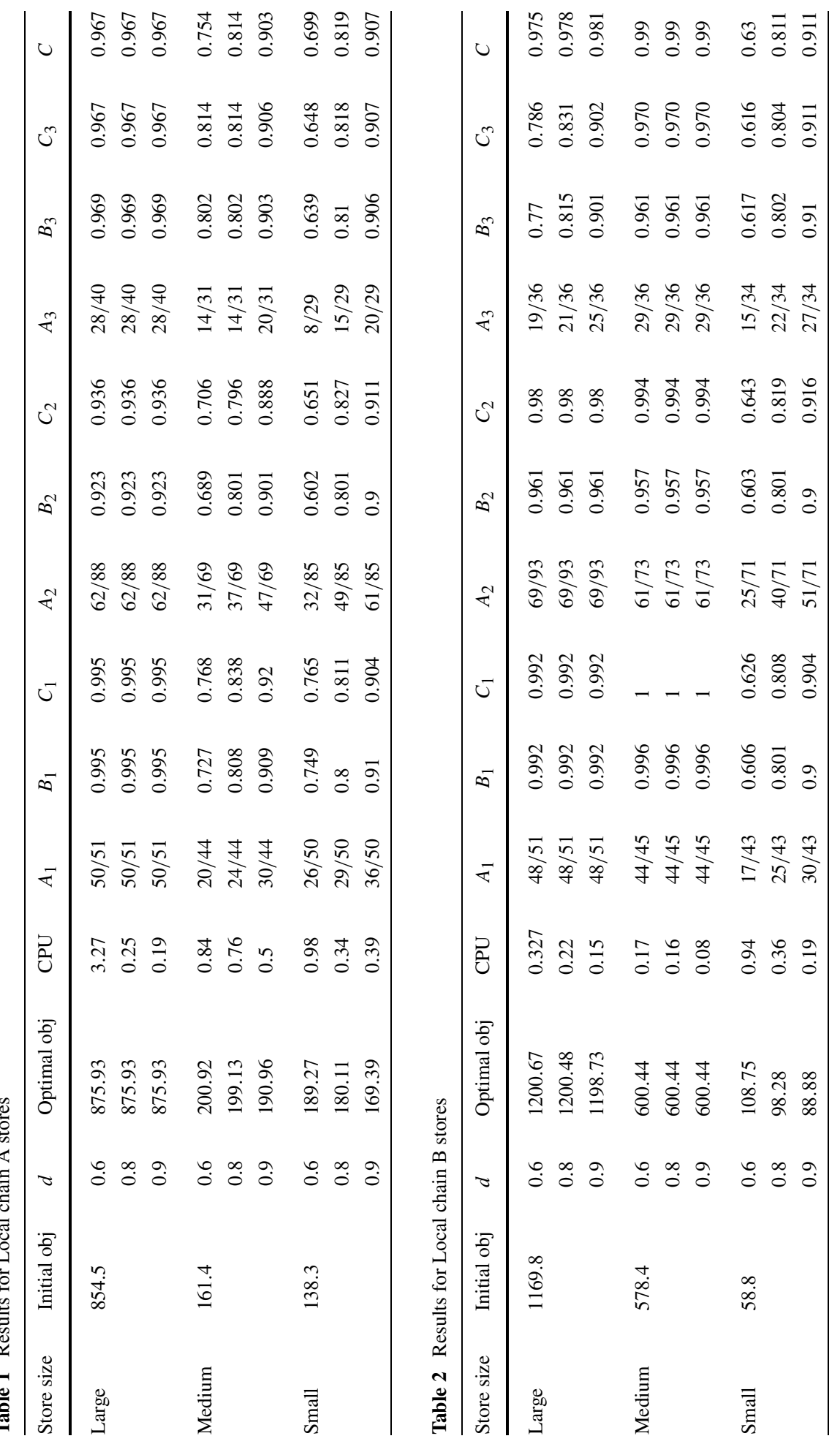


elimination. The column "CPU" gives the solution time of the linearized model in seconds. The quantities $A_{i}, B_{i}, C_{i}, i=1,2,3$, and $C$ are defined as follows:

$$
A_{i}=\frac{\sum_{j} x_{i j}}{N_{i}}, \quad B_{i}=\frac{\sum_{j} q_{i j} x_{i j}}{\sum_{j} q_{i j}}, \quad C_{i}=\frac{\sum_{j} p_{i j} q_{i j} x_{i j}}{\sum_{j} p_{i j} q_{i j}} \quad \text { and } \quad C=\frac{\sum_{i j} p_{i j} q_{i j} x_{i j}}{\sum_{i j} p_{i j} q_{i j}} .
$$

The quantity $A_{i}$ represents the ratio of the number of SKUs kept on the shelf after elimination to the total number of SKUs initially on shelf in category $i$ (SKU retained ratio). Similarly, we use $B_{i}$ to denote the ratio of sales volume from the SKUs kept on the shelf to the total sales volume initially in category $i$ (volume retained ratio). In $C_{i}$ we record the ratio of the profit due to the SKUs kept on the shelf to the total profit initially from category $i$ (value retained ratio). Finally, $C$ is used to quantify the total profit ratio across all categories (overall value retained ratio). Note that the profits involved in the ratios $C_{i}$ and $C$ do not include the SKU keeping costs.

The reader should note that $d$ imposes a lower bound on the volume retained ratio at each category $\left(B_{i}\right)$. For the Local chain A large store, the value of $d$ does not affect the results. In the Local chain A medium and small stores, we observe-based on $A_{i}$ values-decreases in the eliminated SKUs in all categories by an approximately equal factor as the minimum conservation ratio increases. In a more stringent elimination effort (lower values of $d$ ) larger number of SKUs from lower quality categories are removed compared to the premium category. This result agrees with the intuition that higher quality, higher price SKUs resist elimination better than lower quality, lower price ones since the former have potentially a larger contribution to the total profitability of the store. In the local chain B's large and medium stores the minimum conservation ratio $d$ has little or no effect on elimination. In cases the model suggests a considerable elimination when it is not tightly constrained by $d$ (when $d=0.6$ ), the number of eliminated SKUs significantly decreases as $d$ increases. In the small store, a more discernible elimination takes place in all categories while it is slightly more pronounced in the middle category.

We observe that the model advocates a sizable elimination in the small stores, an indication of poor shelf management practices in these stores. The local chains seem to have a tendency to cram too many SKUs even when the space or demand patterns do not justify it. It seems advisable that smaller stores get a handle on profitability increases by keeping a reduced assortment where the reduction should be more severe in the lower quality and lower price categories. In other words, avoiding product pollution seems to be particularly beneficial for smaller stores. This observation is also supported by a more marked profit increase due to elimination in the smaller stores.

It is interesting that the optimal solution of the model suggests that there is a consistent ascendant ordering in the SKUs retained, volume retained and value retained at each category. The model tries to keep the SKUs that contribute a larger share of the original profit while performing elimination. In order to achieve this, it has to conserve SKUs contributing more to the sales volume as well—although to a lesser extent-since sales volume is one of the two determinants of profit along with the price. The SKUs retained trails the previous two measures, since in order to maximize profit one has to keep a certain number of SKUs, but eliminate SKUs with limited contribution to the profit.

Experiment 2 The impact of $c$. In Figs. 1 and 2 we illustrate the impact of the cost parameter $c$ on the elimination of SKUs. We select Local chain A Large store as our test store. In Fig. 1 we report the overall SKU retained ratios $\left(\frac{\sum_{i j} x_{i j}}{\sum_{i} N_{i}}\right)$ achieved by the results of our model at different values of minimum conservation ratios and increasing $c$ from 0 to 2 in increments 


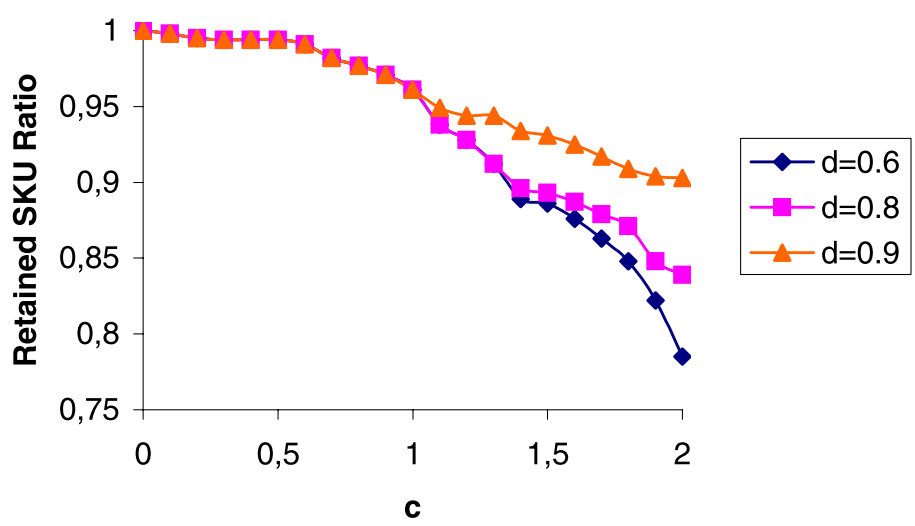

Fig. 1 Overall SKU retained ratio versus $c$

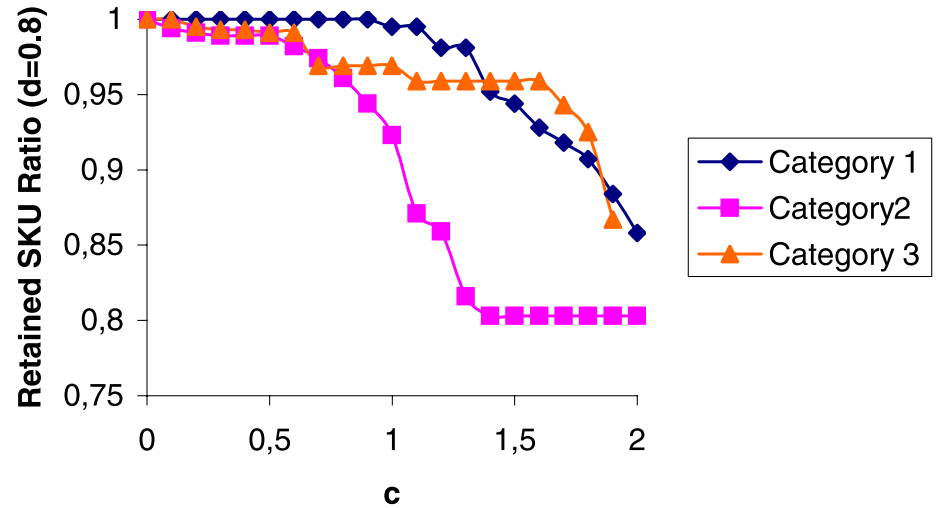

Fig. 2 SKU retained ratio for all three categories versus $c$ at $d=0.8$

of 0.1. As expected, the larger values of $c$, the cost of keeping SKUs on the shelf, lead to increased elimination patterns. In Fig. 2 we report SKU retained ratio in all three categories for the value of $d$ fixed to 0.8 . Interestingly, the middle category SKUs seem to be more susceptible to sharper elimination as $c$ increases than the other categories. Apparently, the model sheds more SKUs from the middle category than from the extreme categories as the cost of keeping SKUs on the shelf goes up. Similar observations are made when the value of $d$ is 0.6 or 0.9 .

Experiment 3 The impact of $s$. While we adopted the value of $s=0.42$ in our previous experiments, we tested the sensitivity of the results to different values of $s$ ranging from 0.35 to 0.5 in increments of 0.1 using the Local chain A large store for illustration. Figure 3 summarizes the results of this experiment at $d=0.8$. An observation similar to that made in Experiment 2 prevails here. The middle category SKUs are clearly more sensitive to perturbations in the substitution ratio $s$ than the extreme categories. The changing values of $s$ do not seem to affect much the high quality and low quality categories. This reflects the existence of an abundance of SKUs exhibiting a wide spectrum of profitability profile, which responds to increases in the substitution ratio by gradually enlarging the set of delisted 


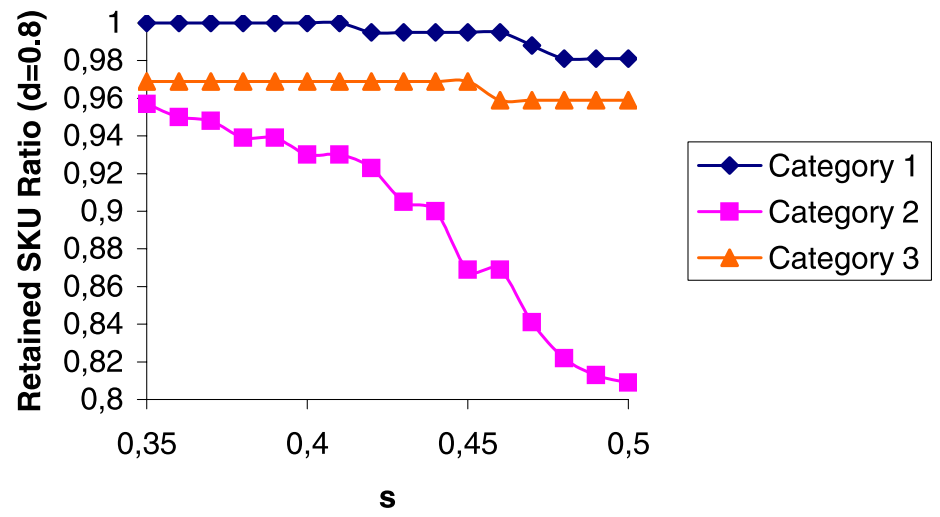

Fig. 3 Retained SKU ratio versus $s$ at $d=0.8$

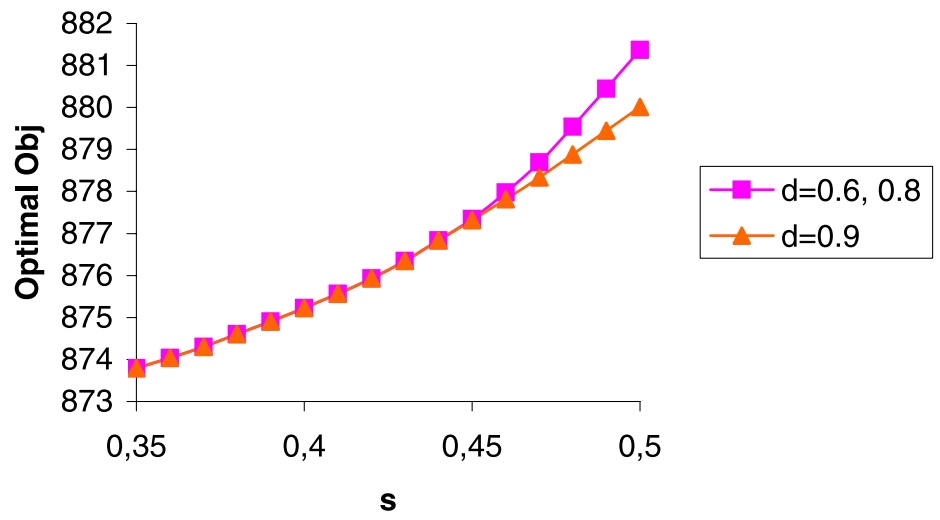

Fig. 4 Optimal objective function value versus $s$

SKUs. It is to be expected that the low quality category does not respond to increased substitution ratio, since the range of prices - and thereby profitability-is rather limited in this category and that does not create an opportunity for capturing profit by substitution. Similar observations are made when the value of $d$ is 0.6 or 0.9 .

The impact of the substitution ratio on the optimal objective function value for different values of $d$ in Fig. 4 indicates a convex increasing curve. As the substitution ratio increases, the change in the optimal objective value gets larger. This impact is less pronounced at higher minimum conservation ratios, i.e., less severe elimination, as expected.

\section{Conclusion}

In this paper we developed a practical optimization model that improves the product mix on the shelves of a retailer, and thus reduces product pollution. We applied the proposed model to two local supermarket chains using proprietary data disclosed to us by the chain managers. This project was undertaken upon the request of an FMCG conglomerate that supplies these-as well as others-retail channels. Their motivation to initiate such an endeavor 
was to persuade the local supermarket chains that a limited assortment would benefit their profitability, while simultaneously solving the visibility problems faced by the market leader products of the FMCG company. To continuously guide the chain manager on the correct assortment, they were asking for a simple and practical tool. We developed such a tool based on the model presented in this paper. To demonstrate the usefulness of our tool, we decided to first focus on the highly problematic shampoo class. Our results that were summarized in Sect. 4 revealed that a judicious elimination based on our model has the potential for a significant increase in profitability. The FMCG company as well as the studied supermarket management are in the process of incorporating this decision support tool into their operations.

\section{References}

Bai, R., \& Kendall, G. (2005). An investigation of automated planograms using a simulated annealing based hyper-heuristics. In T. Ibaraki, K. Nonobe, \& M. Yagiura (Eds.), Operations Research/Computer Science Interfaces Series: Vol. 32. Metaheuristics: Progress as Real Problem Solvers (pp. 87-108). Boston: Springer.

Bai, R., \& Kendall, G. (2008). A model for fresh produce shelf space allocation and inventory management with freshness condition dependent demand. INFORMS Journal on Computing, 20(1), 78-85.

Bai, R., Burke, E. K., \& Kendall, G. (2007). Heuristic, meta-heuristic and hyper-heuristic approaches for fresh produce inventory control and shelf space allocation. Journal of the Operational Research Society. doi:10.1057/palgrave.jors.2602463.

Borin, N., Farris, P. W., \& Freeland, J. R. (1994). A model for determining retail product category assortment and shelf space allocation. Decision Sciences, 25(3), 359-384.

Cachon, G. P., Terwiesch, C., \& Xu, Y. (2005). Retail assortment planning in the presence of consumer search. Manufacturing and Service Operations Management, 7(4), 330-346.

Corsten, D., \& Gruen, T. (2003). Desperately seeking shelf availability: an examination of the extent, the causes, and the efforts to address retail out-of-stocks. International Journal of Retail and Distribution Management, 31, 605-617.

Corstjens, M., \& Doyle, P. (1981). A model for optimizing retail space allocations. Management Science, 27, $822-833$.

Kök, A. G., \& Fisher, M. L. (2006). Demand estimation and assortment optimization under substitution: Methodology and application. Operations Research, 55, 1001-1021.

Kök, A. G., Fisher, M. L., \& Vaidyanathan, R. (2006). Assortment planning: review of literature and industry practice. In N. Agrawal \& S. Smith (Eds.), Retail supply chain management. Amsterdam: Kluwer.

Kurtuluş, M., \& Toktay, L. B. (2005). Category captainship: who wins, who loses? Available at SSRN: http:// ssrn.com/abstract=934970.

Mahajan, S., \& van Ryzin, G. J. (1998). Retail inventories and consumer choice. In S. Tayur, et al. (Eds.), Quantitative methods in supply chain management. Amsterdam: Kluwer.

Mahajan, S., \& van Ryzin, G. (2001). Stocking retail assortments under dynamic consumer substitution. Operations Research, 49, 334-351.

Netessine, S., \& Rudi, N. (2003). Centralized and competitive inventory models with demand substitution. Operations Research, 51, 329-335.

Rajaram, K. (2001). Assortment planning in fashion retailing: methodology, planning and analysis. European Journal of Operational Research, 129, 186-208.

Schaible, S. (1977). A note on the sum of a linear and linear-fractional function. Naval Research Logistics Quarterly, 24, 691-693.

Smith, S. A., \& Agrawal, N. (2000). Management of multi-item retail inventory systems with demand substitution. Operations Research, 48, 50-64.

Urban, T. L. (1998). An inventory-theoretic approach to product assortment and shelf space allocation. Journal of Retailing, 74, 15-35.

van Ryzin, G., \& Mahajan, S. (1999). On the relationship between inventory costs and variety benefits in retail assortments. Management Science, 45, 1496-1509.

Yang, M.-H. (2001). An efficient algorithm to allocate shelf space. European Journal of Operational Research, 60, 309-317.

Yang, M.-H., \& Chen, W. C. (1999). A study on shelf space allocation and management. International Journal of Production Economics, 60, 309-317. 